\title{
The Construction of Mountain Bike Athletes' Performance Measurement Index System Based on Multi-Source Information Fusion
}

\author{
Qingbin Yang \\ School of Leisure Sports, Chengdu Sport University, Chengdu 614000, Sichuan, China \\ Correspondence should be addressed to Qingbin Yang; yangqingbin@cdsu.edu.cn
}

Received 25 December 2021; Revised 17 January 2022; Accepted 14 February 2022; Published 7 March 2022

Academic Editor: Hasan Ali Khattak

Copyright (c) 2022 Qingbin Yang. This is an open access article distributed under the Creative Commons Attribution License, which permits unrestricted use, distribution, and reproduction in any medium, provided the original work is properly cited.

\begin{abstract}
Mountain biking is a recognized fitness sport. Therefore, studying the performance measurement system of mountain biking athletes is an important way to supervise and realize scientific training. At present, many views on the issue of function measurement are somewhat divergent. The only research is limited to the discussion of function components, which lacks systemicity, and there is little research on the construction of the function measurement index system. Combining the practice of mountain bike training, the article conducts a more detailed research on the construction of performance measurement index system, focusing on what to do and the principles to be followed. The article takes a total of 28 mountain bikers from a cycling team as the research object, and uses the literature review method, expert consultation method, and functional test mathematical statistics method to analyze the obtained data and improve the data sorting table. On this basis, statistics create descriptive statistics of the data. Experiments show that if the ankle joints and flexors and extensors of mountain bikers are tested at an angular velocity of $60 \%$ s and $180 \%$ s, the peak moment flexion-extension ratio is $20 \%-36 \%$, hemoglobin rises within $12-24 \mathrm{~h}$, creatine kinase recovers within $36-72 \mathrm{~h}$, and blood urea nitrogen recovers within $24-36 \mathrm{~h}$.
\end{abstract}

\section{Introduction}

Mountain biking is a cyclical physical fitness event. It is a competitive sports event that uses equipment to do work. Its power comes from the contraction of the human lower limb muscles and the process of active pedaling and stretching. When pedaling and lifting of the lower limbs, the flexibility, extension, and angle changes of the knee and ankle joints of the lower limbs are the main sources of power. Mountain bike athletes have more comprehensive requirements for muscle strength than other sports, covering all aspects of absolute strength, explosive power, and endurance. Strength is the direct factor that determines speed. In cycling, strength quality is mainly manifested as the ability of lower limb muscles to overcome mechanical resistance, wind resistance, and ground deformation during pedaling and pulling. The high frequency pedaling operation of mountain bikers relies on the tightly coordinated and alternate contraction of the knee and lower extremity pelvic muscles. It achieves riding efficiency in heavy-load training and competitions. Increasing the strength of mountain bikers is directly related to the performance of this sport and also helps athletes master and use correct technical movements. Athletes and coaches work hard to improve their riding muscles, knee flexors, and ankle agility. The specific strength training time is greater than the comprehensive training time. However, it is not known how the training ratio of the main muscle groups of each joint is organized, to what extent, and so on, and there is no reliable reference standard. Muscle strength is the foundation of athletes' ability and the key to improving athletes' performance. It is very important to determine and evaluate the athlete's muscle strength. Here, it is of great significance to the research of athletes of different sports levels in the same sport. Because athletes in the same sport have a certain main working muscle group, by testing their working muscle groups, test indicators such as peak torque 
value, relative peak torque value, and flexion-extension ratio of the main joint flexor and extensor muscle groups of excellent athletes and ordinary athletes of this sport can be obtained, and one can see the difference between outstanding athletes and ordinary athletes. In the training, excellent athletes are used as a reference to carry out targeted training of ordinary athletes' muscle strength, and at the same time, to further improve the technical deformation caused by insufficient local strength, so as to achieve a multiplier training effect.

Experts at home and abroad also have a lot of research on the construction of multi-source information fusion for the construction of the athlete's skill measurement index system. $\mathrm{Lu}$ C is based on Dempster Shafer (D-S) evidence theory, using the three-level signal of pump, hydraulic power system, and hydraulic drive system to use the multi-source information fusion fault diagnosis method. The feature vectors of these three levels are extracted into three bodies of evidence (BOEs), and the fuzzy membership function is used to construct the basic probability assignment (BPA) of the three BOEs [1]. Zhang C Q assessed the mindfulness that reflects the current attention, awareness and acceptance in the sports environment. Based on previous literature, existing scales, interviews, and feedback with athletes, coaches, and mindfulness experts, an initial project library was generated. Preliminary support for AMQ3 factor structure was established through exploratory factor analysis, and cross-validation was carried out through confirmatory factor analysis [2]. Kurtz J D believes that preparticipation screening is necessary for athletes, but there is still no consensus on the most effective and efficient way to achieve this goal. Most clinical strategies are based on retrospective case series. By applying the standards of the European Society of Cardiology and Seattle, ECG seems to have the lowest false-positive rate in identifying potentially dangerous cardiac abnormalities in athletes [3]. Gallagher J applied pre-defined inclusion and exclusion criteria, and included papers on performance evaluation indicators related to disease, injury, or related diseases. Using the report checklist of adult elite athletes to assess the effectiveness of qualified outcome measures [4]. Weiss L J believes that mountain bikers' rotator cuff injuries can range from contusions and tendinopathy to full-thickness tears. This requires a comprehensive assessment to determine the extent of the injury and an appropriate care plan. Management strategies can range from rehabilitation to surgical intervention, and are guided by the size of the tear, season time, exercise, performance limitations, and the presence of accompanying pathology [5]. Konings $\mathrm{M} \mathrm{J}$ believes that the variability of the finish time of different races within athletes increases with the length of the race $(500 \mathrm{~m}: \mathrm{CV}=1.6 \%$; $1000 \mathrm{~m}: \mathrm{CV}=2.8 \% ; 1500 \mathrm{~m}: \mathrm{CV}=4.1 \%)$. The main reason is that in the initial stages of the $1000 \mathrm{~m}(3.3-6.9 \%)$ and $1500 \mathrm{~m}$ (8.7-12.2\%) races, the race differences among athletes are higher. In these early stages, the intra-match variability of $1000 \mathrm{~m}(1.1-1.4 \%)$ and $1500 \mathrm{~m}(1.3-2.8 \%)$ matches is relatively low [6].

This paper studies the strength characteristics of the pelvic floor muscles of mountain bikers by stretching the knees, ankles, and hands of mountain bikers with different training levels, combined with professional technical analysis and strives to provide a reliable reference for athletes and checks the difference in the degree of preparation of athletes in different isokinetic tests.. Check the difference in the degree of preparation of athletes in different isokinetic tests. Understanding the impact of muscle strength training quality on coaches and athletes can more effectively improve athlete performance, prevent sports injuries, and provide a scientific basis for the scientific selection and analysis of training athletes' muscle strength [7]. Based on the multisource, heterogeneous, and incomplete characteristics of data collected by multi-source sensor networks, this article examines multi-source information synthesis technology, uses effective synthesis technology to provide accurate information about observable events, and roughly analyzes and tracks trends. Provide historical event data to predict current events, and provide coaches and athletes with accurate information and a basis for judgment in training decisions. Therefore, the research and implementation of multi-touch sensor network software and volume analysis software has important significance and broad prospects for mountain bikers [8].

\section{Multi-Source Information Fusion Related Technologies}

According to the level of data abstraction in the information fusion system, fusion can be divided into three levels: datalevel fusion (also called pixel-level fusion), feature-level fusion, and resolution-level fusion. The structure of the fulllevel fusion processing is divided into:

(1) Data-level mixing: The structure of the data layer synthesis is to perform the original combination of the monitoring data of the same type of sensors, so as to extract the data attributes, and finally identify and judge. The premise of combining data levels is that the sensor types must be the same. Only in this way can the data levels be synthesized; if two or more types of sensors are available, they can only be mixed at higher levels, that is, a combination of character levels or a combination of decision-making levels. In the data-level synthesis process, the data obtained by the synthesis center has the characteristics of large data volume and high accuracy, but it has higher requirements for the computing power and speed of the system and network communication [9]. Since this is the lowest level of synthesis, the advantage of directly processing the original data is that most of the original data are retained. Compared to the other two levels of processing, the information quality is unparalleled, without any data loss. But this method has great limitations: One is that it can only process one or more sensors of the same type, and the compatibility of the sensors must be ensured. The other is that a large amount of mixed raw data needs to be processed comprehensively. Therefore, the requirements for communication bandwidth are 


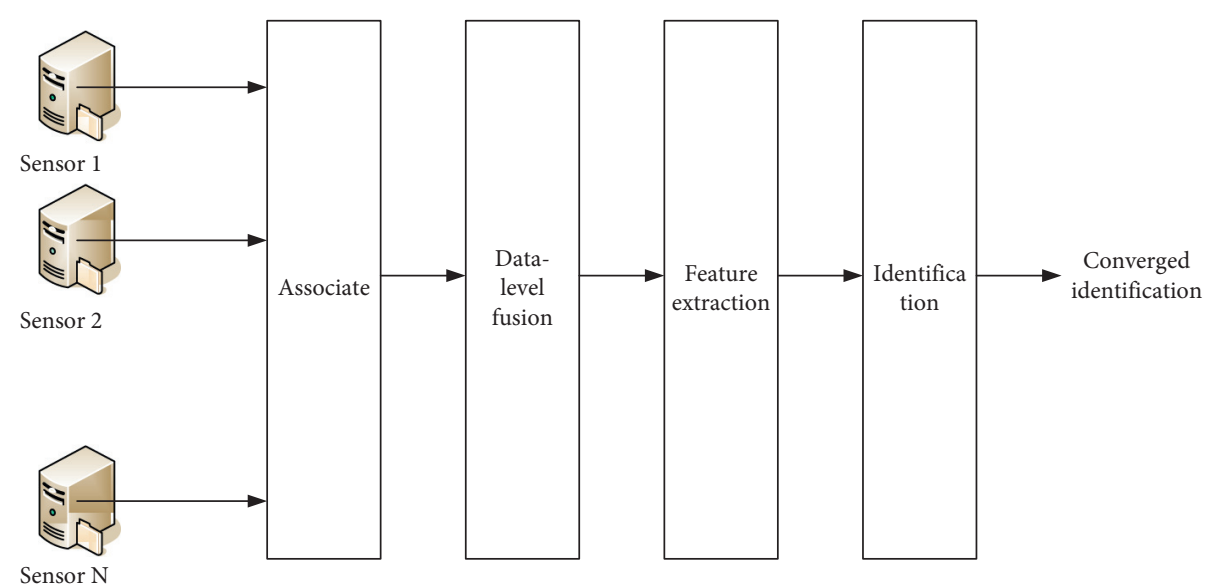

FIgURE 1: Data-level fusion.

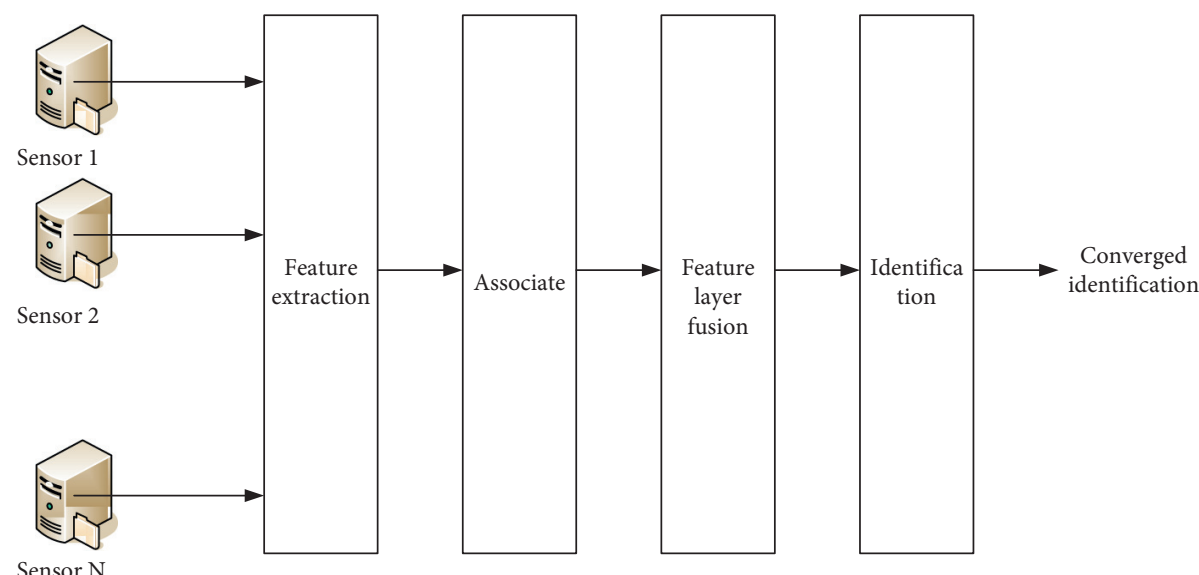

FIgURE 2: Decision-level fusion.

high, and the computational cost is high. The requirements for calculation speed and memory capacity are higher than the other two mixed levels, and the data transmitted through the sensor is incomplete, easily distorted, and unstable (Figure 1).

(2) Feature-level synthesis attributes: The structure of feature-level synthesis is shown in Figure 2. The combination of feature levels belongs to spatial integration and can be roughly divided into two categories: a combination of target character information and a combination of target status information. Each sensor in the feature-level synthesis structure has the function of extracting features from the monitoring data. Each sensor receives a separate part of the monitoring data, and then submits it to the synthesis center for synthesis processing, and finally provides the obtained results for quality evaluation. The characteristic of the fusion feature is that it can be processed in real time, the original information is less lost, and it can provide direct data for the fusion decision. Eliminating the attributes in the data will reduce the total amount of data transmission, thereby reducing communication bandwidth and speeding up processing [10].

Feature-level fusion considers the characteristics of datalevel fusion and decision-level fusion. Its advantages are: feature extraction of original information, compression of data, reduction of transmission bandwidth, and low requirements for communication bandwidth; the disadvantage is compared to data-level fusion. Obtaining features at this level will lead to the loss of some information, thereby reducing the accuracy of the data, as shown in Figure 2.

Commonly used fusion algorithms in feature-level fusion include basic Kalman filtering algorithm, joint probabilistic data connection, multiple hypotheses, interactive model methods, and sequential processing theory. The target information fusion feature mainly uses clustering method, artificial neural network, K-order nearest neighbor algorithm, etc. [11].

Figure 3 shows the process in which the sensor removes and recognizes the characteristics of the monitored data, and then transmits the recognition results to the fusion processing center. The synthesis processing center makes the final decision based on the processing status and identifies all local synthesis sensors. The integration of the decision- 


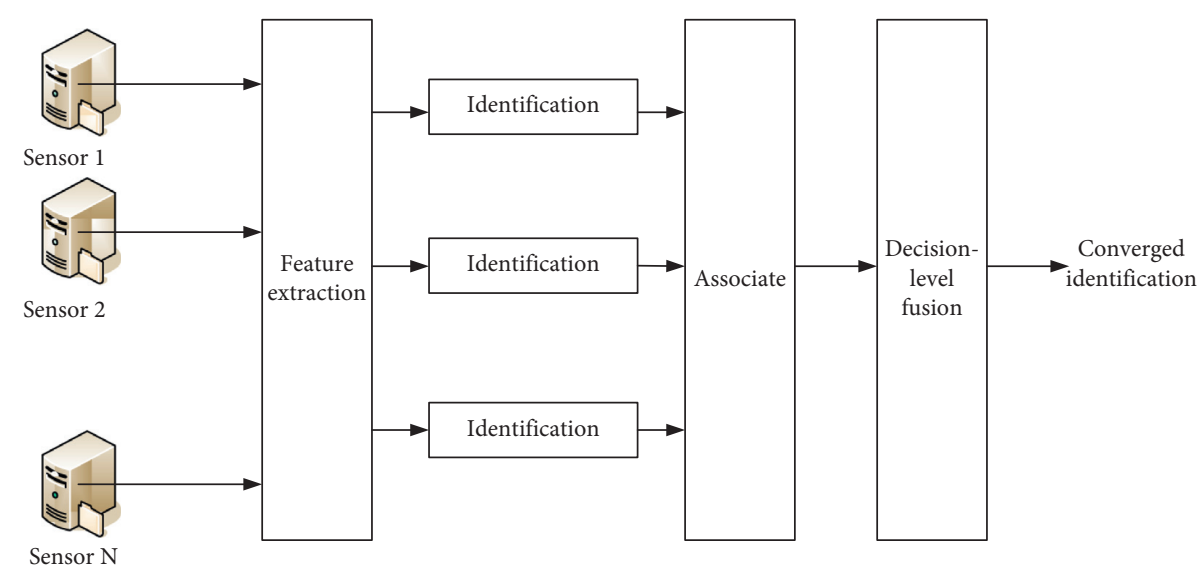

FIgURe 3: Decision-level fusion.

making level is directly related to the specific decisionmaking goals, and the result directly affects the decisionmaking level. The advantages of the synthesis decision layer are: It can integrate different types of sensors, the amount of information processing in the synthesis process is small, the real-time performance is high, and the ability to resist tampering is strong. The disadvantage is that due to the multi-level processing of sensor data synthesis, information loss is more serious, which reduces the accuracy of the synthesis result. Fusion algorithms commonly used in decision-level synthesis include: Bayesian estimation, D-S proof system theory, indefinite set theory, etc. [12]. The comparison of the three attribute-level comprehensive attributes is shown in Table 1:

\subsection{The Idea of Multi-Source Information Compound Fusion} Method Based on Context Weight. From related literature and experimental research, it is found that there is no perfect fusion algorithm, and satisfactory results can be obtained in various situations. Different fusion algorithms have their own characteristics, and some are suitable for information fusion of static targets; some have high time complexity, large amount of calculation, and poor real-time performance, which are not suitable for real-time information fusion; some of the prerequisites required for fusion are relatively harsh and not easy to achieve, which leads to a large deviation between the fusion decision information and the result. In addition, if the sensor is used as a fusion data source, the monitoring performance of different types of sensors is affected by the type of object being monitored, the geographic location of the sensor, the weather, and the sensor itself, which may lead to deviations or errors in the monitoring data [13]. If only one fusion algorithm is used for fusion, the result is prone to large errors or even errors. In response to the above problems, this paper proposes a multisource information composite fusion framework based on context weights, as shown in Figure 4:

The main purpose of multi-source information composite fusion is to overcome the fusion errors caused by the shortcomings or limitations of a single fusion method, and to improve the accuracy of the fusion system. The core idea of the composite fusion framework is to use multiple fusion methods to perform parallel fusion calculations on the monitoring data of the same event, and then use the weighted average of the fusion results of each fusion algorithm as the final fusion result. First, according to the context in which the monitoring data is located, combined with the data performance of the fusion algorithm in processing this context, the fusion result is assigned a corresponding weight (The fusion algorithm is given a higher weight if the data fusion effect in this context is good, otherwise the weight assigned is low). Then, the multiple parallel fusion results are weighted and summed to obtain the final fusion result, and then compared with the set threshold to give the judgment result. The specific process is shown in Figure 5 [14]. The composite fusion framework can utilize the advantages of each fusion algorithm and reduce the influence of the fusion algorithm on the fusion result due to its own shortcomings.

2.2. Sensor Accuracy Weighted Fusion. In real applications, in order to get more accurate results, multiple sensors can be used to measure the same target. However, the different physical characteristics of sensors will inevitably lead to differences in accuracy between sensors. The direct equal weight of the usually obtained sensor measurement data can lead to inaccurate results. For this reason, it can consider using sensor accuracy to measure data. Perform processing to obtain more accurate measurement values. The specific process of the source of the accuracy of the measurement sensor is as follows:

First of all, it is necessary to consider two different sensors measuring the same target at time $k$, and the measured values are, respectively:

$$
\begin{aligned}
& z_{k, 1}=x_{k}+y_{k, 1}, \\
& z_{k, 2}=x_{k}+y_{k, 2} .
\end{aligned}
$$

Since the estimated value is an unbiased estimate of $\mathrm{xk}$, then

$$
\widehat{x}_{k}=w_{k, 1} z_{k, 1}+w_{k, 2} z_{k, 2} \text {. }
$$


TABLE 1: Comparison of attributes-level fusion characteristics.

\begin{tabular}{|c|c|c|c|c|}
\hline & Data-level fusion & Feature-level fusion & Decision-level fusion & Numerical value \\
\hline Information processing capacity & Maximum & Smaller & The smallest & 15 \\
\hline Information loss & The smallest & Smaller & Maximum & 5 \\
\hline Anti-interference ability & The smallest & Smaller & The smallest & 25 \\
\hline Fault tolerance & Worst & Relatively poor & Better & 13 \\
\hline Fusion algorithm difficulty & Difficult & Medium & Easy & 9 \\
\hline Pre-fusion processing & The smallest & Medium & Maximum & 2 \\
\hline Fusion performance & Most & Medium & Difference & 21 \\
\hline Sensor dependence & Big & Medium & Small & 4 \\
\hline
\end{tabular}

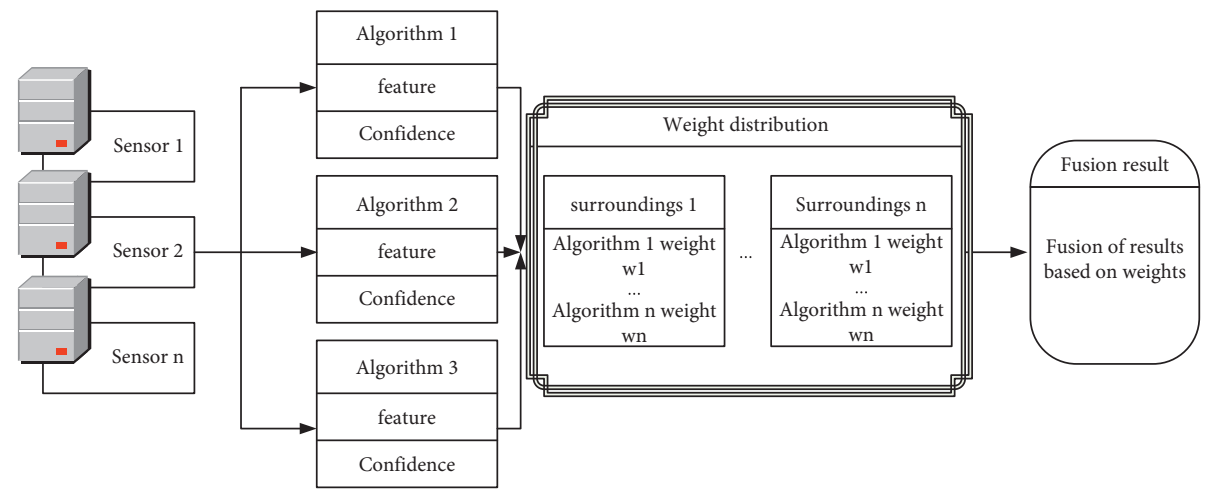

FIGURE 4: Multi-source information composite fusion framework diagram based on context weight.

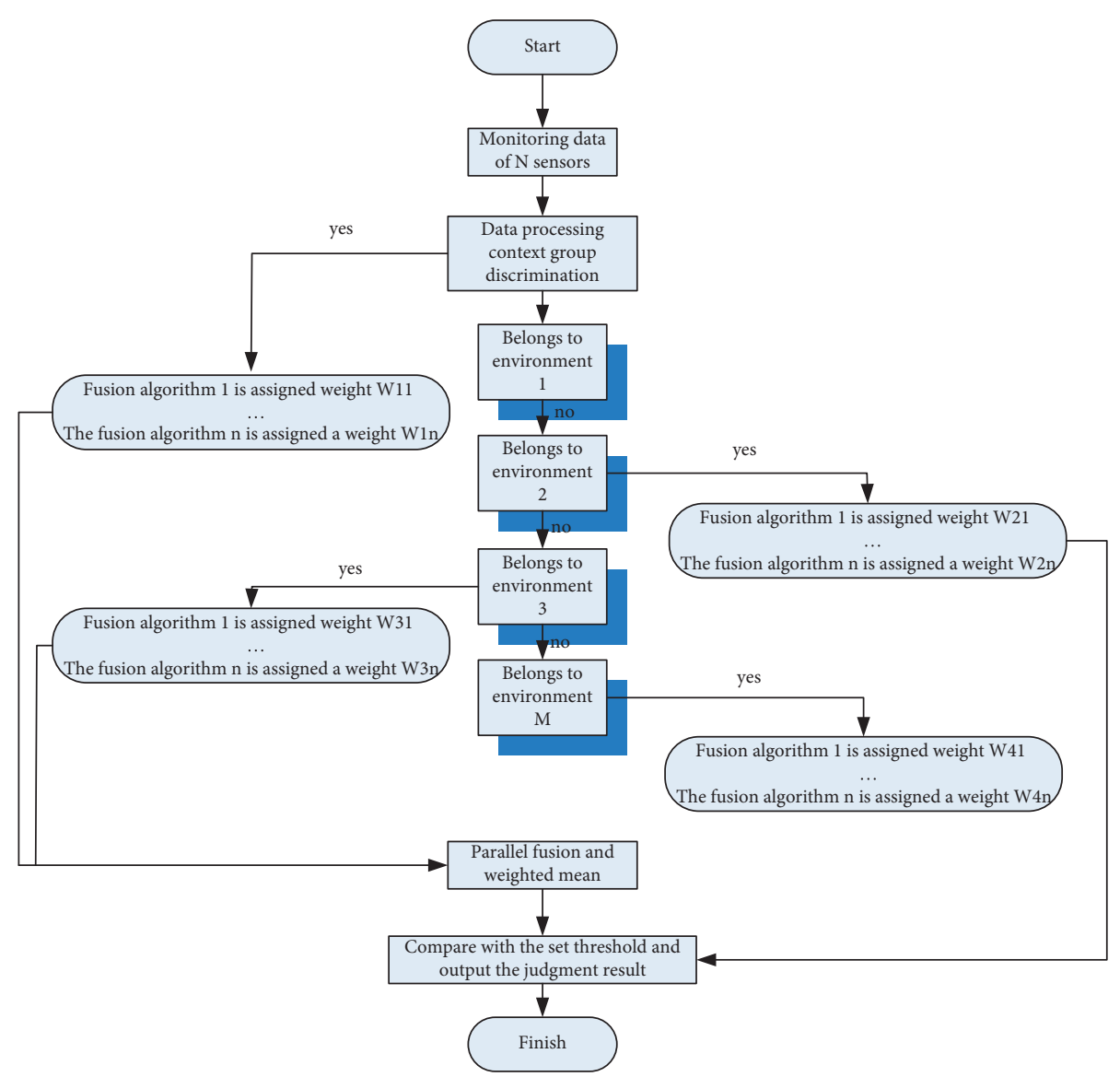

FIGURE 5: Flow chart of multi-source information compound fusion based on context weight. 
$w_{k 1}$ and $w_{k 2}$ represent the weight of the measured value of each sensor. At this time, the estimated error is

$$
\widehat{x}_{k}=x_{k}-\bar{x}_{k} \text {. }
$$

Use the cost function J to represent the root mean square error of $\hat{x}_{k}$, then

$$
\begin{aligned}
J & =E\left(\bar{x}_{k}^{2}\right) \\
& =E\left\{\left[x_{k}-w_{k, 1}\left(x_{k}+y_{k, 1}\right)-w_{k, 2}\left(x_{k}+y_{k, 2}\right)\right]^{2}\right\} .
\end{aligned}
$$

Because $\bar{x}_{k}$ is an unbiased estimate of $x_{k}$, we can get

$$
E\left(\hat{x}_{k}\right)=E\left[x_{k}-w_{k, 1}\left(x_{k}+y_{k, 1}\right)-w_{k, 2}\left(x_{k}+y_{k, 2}\right)\right]=0 \text {. }
$$

It can get

$$
w_{k, 2}=I-w_{k, 1} \text {. }
$$

Where I is the proper-dimensional identity matrix. The cost function $\mathrm{J}$ can be rewritten as follows:

$$
J=E\left[\left(w_{k, 1}\right)^{2}\left(y_{k, 1}\right)^{2}+\left(I-w_{k, 1}\right)^{2}\left(y_{k, 2}\right)^{2}+2\left(w_{k, 1}\right)\left(I-w_{k, 1}\right)\left(y_{k, 1}\right)\left(y_{k, 2}\right)\right] .
$$

In order to find the smallest cost function $\mathrm{J}$, let $\Omega=\left(w_{k, 1}, w_{k, 2}\right)$, and derivate $\Omega$

$$
\frac{\partial J}{\partial \Omega}=0
$$

Three types of Kalman filter implementation structures are based on consistency. Among them, the Kalman singledistribution filtering algorithm has more stable filtering results. By comparing the Kalman canonical filtering algorithm and the central weight fusion, it can be seen that the Kalman canonical filtering algorithm is an equivalent form of intermediate synthesis. Therefore, understanding the Kalman canonical filtering algorithm may be an idea. The summary is as follows: After each sensor node receives the neighboring node data, it executes the Kalman filter algorithm to obtain the estimated value; then, by modifying the ratio of neighboring nodes, accepting consensus recommendations, the final estimated value is obtained. This concept of using Kalman filter defines the Kalman volume filter through a powerful combination of modeling strategy and CKF [15].

Combined with the implementation structure of the consistent Kalman filter algorithm, the update steps of each sensor node $i$ of the volumetric Kalman uniform filtering algorithm are as follows:

$$
\begin{aligned}
J_{i} & =\Theta_{i} \cup\{i\}, \\
z k, i & =\left[z_{k, j_{i 1}}^{T}, z_{k, j_{i 2}}^{T}, \ldots, z_{k, j_{i N}}^{T}\right]^{T}, \\
h k, i & =\left[h_{k, j_{i 1}}^{T}, h_{k, j_{i 2}}^{T}, \ldots, h_{k, j_{i N}}^{T}\right]^{T}, \\
R_{k, j} & =\operatorname{diag}\left[R_{k, j_{i 1}}, R_{k, j_{i 2}}, \ldots, R_{k, j_{i N}}\right] .
\end{aligned}
$$

$j_{i 1}, j_{i 2}, \ldots j_{i N} \in J_{i}, J_{i}$ represent the set of sensor node $i$ itself and the set of communicable neighbors $\Theta \mathrm{i}$, the total number of elements in set $j_{i}$ is $\mathrm{N}$. The remaining parameters are consistent with the volume Kalman filter.

$$
\begin{aligned}
P_{k-1 k-1, i} & =S_{k-1 k-1, i}\left(S_{k-1 k-1, i}\right)^{T}, \\
X_{k-1 k-1, i}^{a} & =S_{k-1 k-1, i} \xi_{a}+\widehat{x}_{k-1 k-1, i}, \\
X_{k k-1, i}^{*, a} & =f_{k-1, i}\left(X_{k-1 k-1, i}^{a}\right), \\
\widehat{x}_{k k-1}, i & =\sum_{a=1}^{L} X_{k k-1, i}^{*, a} L \\
P_{k k-1, i} & =\sum_{a=1}^{L}\left(X_{k k-1, i}^{*, a}\left(X_{k k-1, i}^{*, a}\right)^{T}\right) L-\widehat{x}_{k k-1}\left(\bar{x}_{k k-1, i}\right)^{T}+\Gamma_{k-1} Q_{k-1} \Gamma_{k-1}^{T} .
\end{aligned}
$$

Calculate the predicted value of the measurement $\bar{z}_{k k-1, i}$ 


$$
\begin{aligned}
P_{k k-1, i} & =S_{k k-1, i}\left(s_{k k-1, i}\right)^{T}, \\
X_{k k-1}^{a} & =S_{k k-1} \xi_{a}+\bar{x}_{k k-1, i}, \\
Z_{k k-1, i}^{a} & =h_{k, j}\left(X_{k k-1, i}^{a}\right), \\
\bar{z}_{k k-1, i} & =\sum_{a=1}^{L} Z_{k k-1, i}^{a} L .
\end{aligned}
$$

Centralized CKF has higher accuracy than single-sensor $\mathrm{CKF}$ and improves data reliability. And, it simulates information from all over the world, so that the estimated value of each sensor node gradually increases over time, which helps to eliminate the difference between the readings. The combination of the two not only improves the accuracy of algorithm evaluation but also reduces the analysis bias in the analysis [16].

\section{Research Contents and Methods of Constructing the Index System of Mountain Bike Athletes' Performance Measurement}

3.1. Research Content. Research on the strength characteristics of the knee joint flexors and extensors of male mountain bike athletes.

A study on the strength characteristics of the ankle joint flexors and extensors of male mountain bikers.

3.2. Research Objects. Taking a cycling team and a total of 28 mountain bikers as the research object, the longest period of special training is 8 years and the shortest is 2 years. In order to compare the differences in the abilities of cyclists at different levels, this article divides the subjects into an excellent group and a normal group according to their exercise level. Among them, the excellent group is set as masters and first-level athletes, and the general group is set as secondlevel and third-level athletes. During the test, none of the 28 athletes had significant waist, knee, or ankle injuries. Table 2 shows the basic situation of the research objects.

\subsection{Research Methods}

3.3.1. Experimental Test Method. The test equipment adopts the CYBEX-NORM770 isokinetic strength test and rehabilitation equipment produced by the American CYBEX company. The equipment mainly includes constant velocity power meter head, test chair, test accessories, computer, printer, etc. Through eight movable joints of the human body, 23 related procedures are used to carry out active and passive tests of the strength of 45 major muscle groups such as extension and flexion, internal and external rotation, and adduction and abduction.

Perform dynamic isokinetic centripetal tests on male mountain bikers' flexors and extensors, knee joints, and pelvic extension. According to the maximum output rate, the flexors, knee flexors, and extensors are tested at two different angles: a slower constant speed of $60 \%$ and a faster constant speed of $300^{\circ} / \mathrm{s}$. The test angles for the flexibility and strength of the leg joints are slow with constant speed $60 \%$ and fast with constant speed $180^{\circ} \mathrm{s}$. In the slow and constant speed test of muscle dynamics, the resistance of the limbs to the load is greater. Therefore, it is often used to evaluate the maximum muscle strength. If in the fast and constant speed experiment, the resistance of the limbs to the load is small, and the joint movement speed is fast, so it is often used to measure high-strength muscles [17].

3.4. Condition Control. During the test, the subject's physical condition, mental state, subjective consciousness, body posture, warm-up activities, mastery of movement skills, and other factors have a special impact on the accuracy of the test. Therefore, in order to minimize errors, certain things need to be controlled.

(1) Subject's physical and mental condition: Whether the subject's physical function is normal, whether he or she is sick, and whether he or she has fatigue symptoms. The task is clear for the purpose of the test and whether the mind is calm.

(2) The extent to which the subject enters the working state: whether the subject's general thermal activity before the test and the special thermal activity of the specific muscle group joint test are sufficient.

(3) Athlete's adaptive training: Before taking part in the test formally, the subjects must fully understand the performance of the isokinetic tester, understand its use method, and make the data more accurate.

(4) Disciplinary practice level: Whether it shows the maximum strength required in the formal test.

(5) Pre-tensioning of muscles before work: Pre-tensioning of muscles before work is an important condition for quickly exerting muscle strength and maximizing force. Muscles usually need to enter a pre-tightened state in order to contract forcefully immediately.

\subsection{Test Process.}

(1) Turn on the machine, select the test program code and related parameters, adjust the machine according to the specific test situation, and install the corresponding accessories.

(2) Measure the height and weight of the tester.

(3) Register the tester's name, gender, age, physique, weight, special sports, special training years, highest score, and other relevant data.

Before the test, explain the test essentials to the subjects, so that the subjects will be fully prepared. This is not only conducive to the accuracy of the test but also to avoid muscle strain during the test. According to the characteristics of the sport, choose the test program that is closest to the actual action in the manual. The specific test methods for the knee and ankle joints are as follows. Knee muscle strength test program: Let the subject take a sitting position, 
TABLE 2: Basic situation of research objects.

\begin{tabular}{lccccc}
\hline & $\begin{array}{c}\text { Number of people } \\
\text { (people) }\end{array}$ & $\begin{array}{c}\text { Average age } \\
\text { (years) }\end{array}$ & $\begin{array}{c}\text { Average height } \\
(\mathrm{cm})\end{array}$ & $\begin{array}{c}\text { Average weight } \\
(\mathrm{kg})\end{array}$ & $\begin{array}{c}\text { Average training years } \\
\text { (years) }\end{array}$ \\
\hline $\begin{array}{l}\text { Excellent } \\
\text { group }\end{array}$ & 12 & $20.25 \pm 2.83$ & $176.42 \pm 1.48$ & $76.20+12.10$ & $5.25+2.31$ \\
Normal group & 16 & $19.16 \pm 2.40$ & $173.26 \pm 5.10$ & $72.36+13.80$ & $4.42 \pm 1.15$ \\
Total & 28 & $20.15 \pm 1.08$ & $175.14 \pm 2.35$ & $74.53 \pm 12.85$ & $4.85 \pm 1.52$ \\
\hline
\end{tabular}

fix the upper body and thigh with a wide band, and hold the handles on both sides of the test chair with both hands. The resistance pad connected to the power meter was fixed $3 \mathrm{~cm}$ above the lateral malleolus of the test side, and the rotation axis of the power head was consistent with the knee bone. Weigh the lower limbs first, and the tested muscle strength is corrected by the weight of the lower limbs, and the test range of activities is uniformly set at F02D $10^{\circ}-95^{\circ}$.

(4) Set the relevant parameters of the test according to the subject's personal situation, and divide into two groups of test angular speeds of $60 \%$ and $300 \%$ s. Before the test, let the subjects perform two test exercises, let the subjects feel the test speed and force direction, and then conduct the formal test. First, perform a fast constant velocity test with an angular velocity of $300 \%$, and then perform a slow constant velocity test with an angular velocity of $60 \%$. After each set of speed test is completed, rest for 30 s before doing the next set of speed test [18].

Ankle muscle strength test program: Ask the subjects to lie on their backs and board, the waist and knee joints are fixed as required, the feet are relaxed, and the center of the ankle joint is consistent with the axis of rotation. The other methods are the same as when testing the knee joint, and the angular velocity is tested in two groups $\left(60^{\circ} / \mathrm{s}, 180^{\circ} / \mathrm{s}\right)$. First carry out an angular velocity test of $180 \%$, and then carry out an angular velocity test of $60 \%$ s. After each speed test is completed, rest for 30 s before doing the next speed test.

(5) After the test is completed, save the test results in time, and check whether there is any data loss. Table 3 shows the specific parameters of the test site.

As shown in Table 4 and Figure 6, the high values of joint torque and knee length of the elite group and ordinary mountain bikers decrease with the increase of the test angle. If the measured angular velocity is $60 \%$, the maximum torque of the left and right knee joints and extensor groups of the excellent group and the normal group is very different $(P<0.05)$. There is a big difference between the excellent group of right knee maximum torque and the normal group $(\mathrm{P}<0.01)$. The difference between the right and left knee joints in the general group of athletes was very obvious $(P<0.01)$. When the test angular velocity is $300 \%$, the peak torque of the knee joint and the muscle extension torque of the ordinary group of athletes are significantly different from those of the excellent group $(P>0.05)$ [19].
It can be seen from Table 5 and Figure 7 that the relative peak torque values of the knee flexors and extensors of the male elite group and ordinary mountain bikers decrease with the increase of the test angular velocity. If the angular velocity is tested at $60 \%$ s and $300 \%$, there is no significant difference in the relative peak torque of knee flexion and extension between the elite group and the ordinary group $(P>0.05)$.

As shown in Table 6 and Figure 8, the maximum torque angles of the flexors and knee flexors and extensors of the mountain bike athletes in the excellent group and the ordinary group increased with the increase of the test speed. When the test angular velocity is $60 \%$, there is a big difference between the peak torque of the left and right elbow flexor groups and the maximum torque angle of ordinary athletes $(P<0.01)$. If the test angular velocity is $300^{\circ} / \mathrm{s}$, there is no significant difference between the right and left knee joints and the expansion moment angle between the excellent group and the normal group $(P>0.05)$. The vertex angle of lateral knee flexion may be the same.

It can be seen from Table 7 and Figure 9 that the average power of the knee flexors and extensors of the male elite group and the ordinary group of mountain bike athletes increases with the increase of the test speed. When the test angular velocity was $60 \%$, the average power of the left and right knee joint flexors and extensors was significantly different between the athletes in the excellent group and the ordinary group $(\mathrm{P}<0.01)$. When the test angular velocity was $300 \%$, the average power of the left and right knee joint flexors and extensors was not significantly different between the athletes in the excellent group and the ordinary group $(P>0.05)$.

Table 8 and Figure 10 show the strength test of the knee flexors and extensors of the excellent mountain bike group and the ordinary mountain bike group, as well as the strength and increase of the left and right knees $(P>0.05)$. When the angular velocity of the test is $60 \%$, the relative average strength of the left and right knee flexors and joint extensors of the athletes in the excellent group and the ordinary group are quite different $(\mathrm{P}<0.01)$. If the test angular velocity is $300 \%$, there is no significant difference in the relative average power and extension of the left and right knee flexors between the elite group of athletes and the ordinary group of athletes $(P>0.05)$. The research structure of changes in important hormone parameters (serum testosterone and cortisol) is related to heredity and temperature. Everyone has obvious differences. The transition time is usually more than 15 days, so these differences often vary from person to person [20]. Changes in blood lactic acid are opposite to changes in hormone levels. This only depends on 
TABLE 3: Specific parameter settings of the test site.

\begin{tabular}{lccc}
\hline Joint & Contraction form & Test angular velocity adjustment $\left({ }^{\circ} / \mathrm{s}\right)$ & Repeat times \\
\hline & & $300 \%$ & 14 \\
Knee joint & Bending/stretching & $60 \mathrm{P} / \mathrm{s}$ & 6 \\
& & $180 \%$ & 10 \\
Ankle joint & Sole/Sole & $60 \mathrm{P} / \mathrm{S}$ & 6 \\
\hline
\end{tabular}

TABLE 4: The peak torque values of knee flexors and extensors of male Mountain biker in the excellent and ordinary groups (N.m).

\begin{tabular}{lcccc}
\hline & & $60 \% \mathrm{~s}$ & & $300^{\circ} \mathrm{s}$ \\
& Flexors & Extensors & Flexors & Extensors \\
\hline Excellent group left & $140.52 \pm 14.53$ & $250.13 \pm 29.08$ & $86.40 \pm 12.17$ & $124.30 \pm 16.85$ \\
Normal group left & $126.38 \pm 17.42$ & $218.00 \pm 46.11$ & $88.98 \pm 15.99$ & $135.2 \pm 22.89$ \\
Excellent group on the right & $147.40 \pm 19.65$ & $239.70 \pm 29.44$ & $89.90 \pm 13.33$ & $131.92 \pm 18.25$ \\
Normal group right & $123.2 \pm 19.25$ & $201.6 * 38.56$ & $93.29 \pm 15.36$ & $129.87 \pm l 9.68$ \\
\hline
\end{tabular}
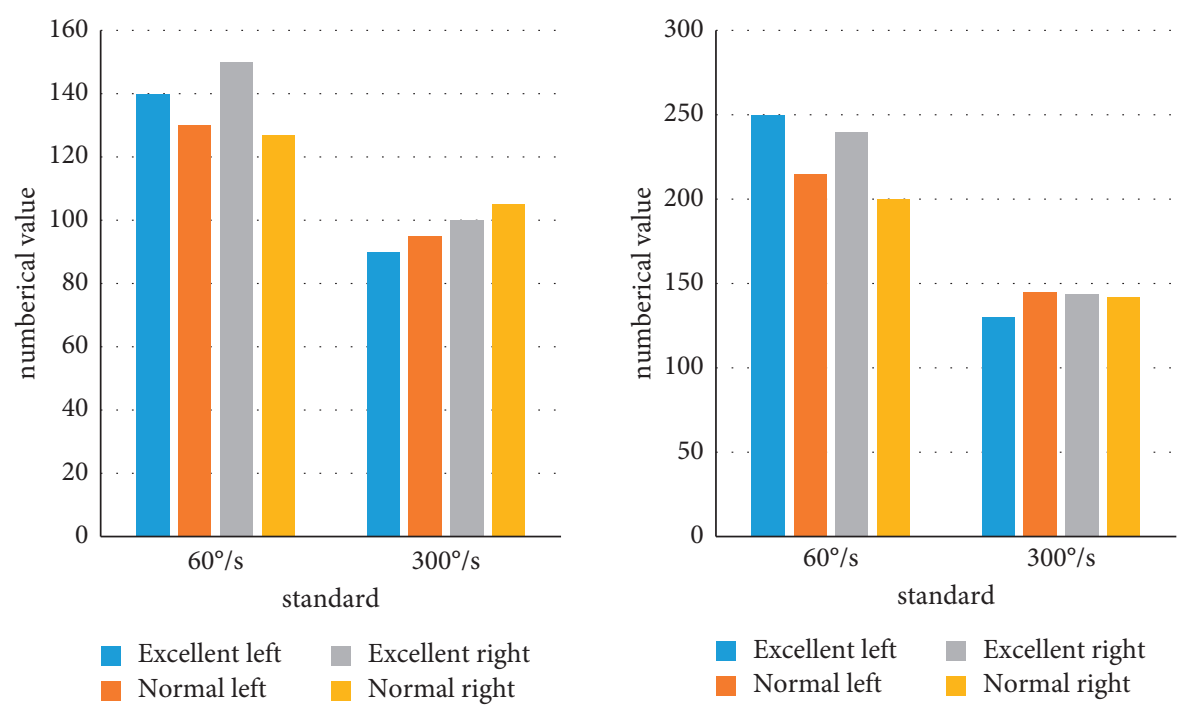

FIgURE 6: The peak torque values of the knee extensor muscles of male Mountain bikers in the excellent and ordinary groups.

TABLE 5: The relative peak moments of the knee flexors and extensors of male cyclists in the excellent and ordinary groups $\left(\mathrm{N}^{*} \mathrm{~m} / \mathrm{kg}\right)$.

\begin{tabular}{lcccr}
\hline & & $60^{\circ} / \mathrm{s}$ & & $300^{\circ} / \mathrm{s}$ \\
& Flexors & Extensors & Flexors & $1.13 \pm 0.19$ \\
Excellent group left & $1.87 \pm 0.19$ & $3.20 \pm 0.33$ & $1.18 \pm 0.21$ & $1.59 \pm 0.19$ \\
Normal group left & $1.69 \pm 0.71$ & $2.99 \pm 0.41$ & $1.15 \pm 0.15$ & $1.78 \pm 0.30$ \\
Excellent group on the right & $1.89 \pm 0.23$ & $3.16 \pm 0.19$ & $1.23 \pm 0.14$ & $1.70 \pm 0.26$ \\
Normal group right & $1.75 \pm 0.25$ & $2.80 \pm 0.27$ & $1.76 \pm 0.23$ \\
\hline
\end{tabular}

the intensity of the training and has nothing to do with other factors, but the change usually occurs within 10 minutes. Through the long-term monitoring of the players, it is also found that the lactic acid level is significantly related to the training and sports of the athletes. In other words, it is difficult for ordinary athletes to accumulate lactic acid after prolonged exercise. The blood content is not high, not more than $12 \mathrm{mmol} / \mathrm{L}$, and it decreases with age. Athletes who exercise a lot are more easily tolerated, reaching more than $14 \mathrm{mmol} / \mathrm{L}$. The maximum content of lactic acid in the blood of athletes is theoretically $4 \mathrm{mmol} / \mathrm{L}$, but there are significant differences in the specific circumstances. It mainly depends on oxygen uptake, cardiovascular function, and lactate value [21]. For athletes whose normal lactic acid level is lower than $2 \mathrm{mmol} / \mathrm{L}$, the highest blood lactic acid level is about $4 \mathrm{mmol} / \mathrm{L}$. It is difficult to determine the threshold of their anaerobic activity, the changes are irregular, and the progress of such athletes to improve their level is slow. Good athletic performance is based on a good functional state, and a good functional state is to achieve good athletic 

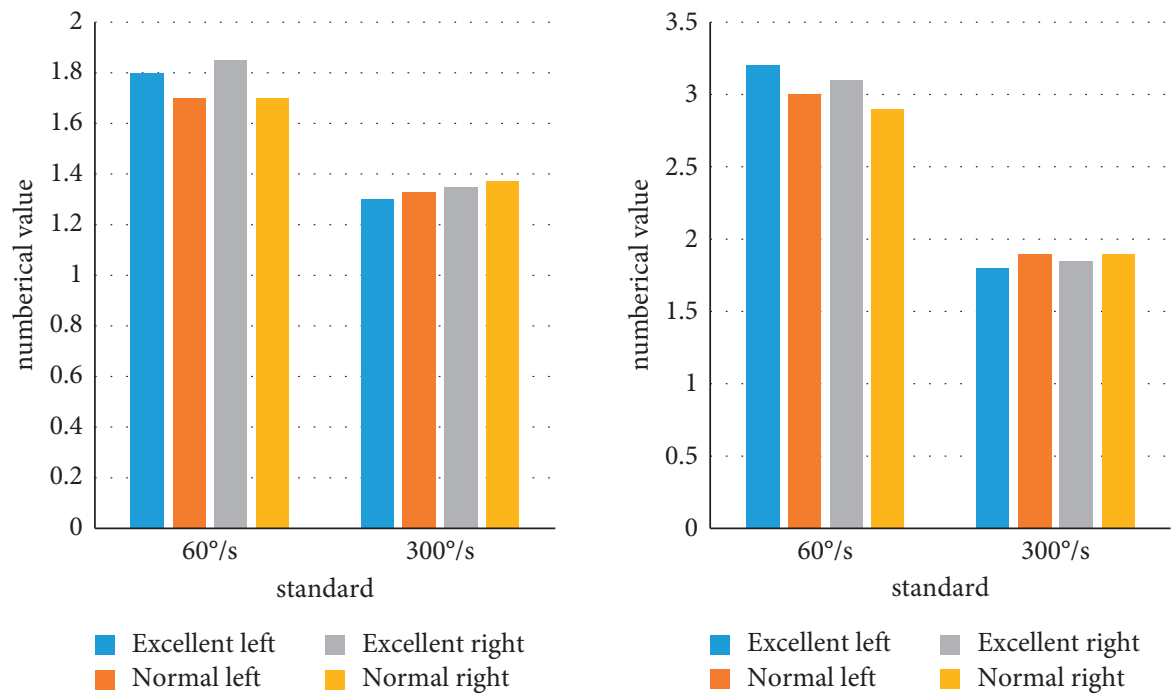

Figure 7: The relative peak torque values of the knee flexors of male Mountain bikers in the excellent group and the ordinary group.

TABLE 6: The peak moment angles of the knee flexors and extensors of male Mountain bikers in the excellent and ordinary groups.

\begin{tabular}{|c|c|c|c|c|}
\hline & \multicolumn{2}{|c|}{$60 \% \mathrm{~s}$} & \multicolumn{2}{|c|}{$300^{\circ} / \mathrm{s}$} \\
\hline & Flexors & Extensors & Flexors & Extensors \\
\hline Excellent group left & $21.11 \pm 7.39$ & $61.60 \pm 6.87$ & $33.65 \pm 8.45$ & $57.40 \pm 5.27$ \\
\hline Normal group left & $25.90 \pm 6.20$ & $56.52 \pm 7.00$ & $28.38 \pm 6.71$ & $58.00 \pm 5.25$ \\
\hline Excellent group on the right & $19.60 \pm 5.50$ & $58.00 \pm 5.90$ & $34.00 \pm 4.06$ & $57.15 \pm 7.87$ \\
\hline Normal group right & $27.08 \pm 6.60$ & $56.65 \pm 5.45$ & $32.30 \pm 6.04$ & $58.08 \pm 5.12$ \\
\hline
\end{tabular}

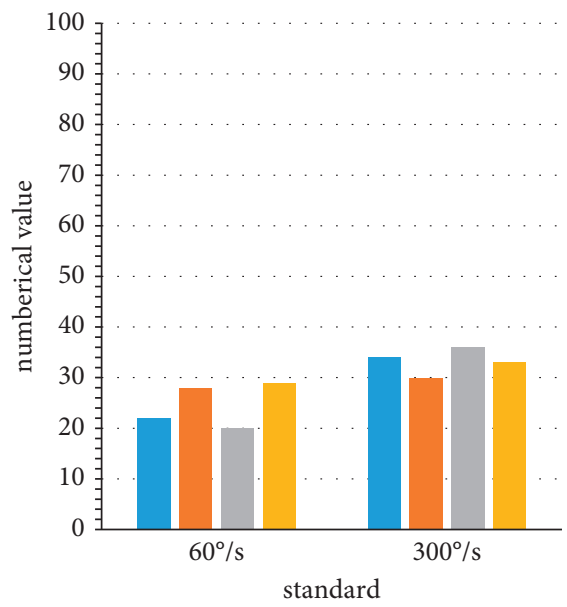

$\begin{array}{ll}\text { Excellent left } & \text { Excellent right } \\ \text { Normal left } & \text { Normal right }\end{array}$

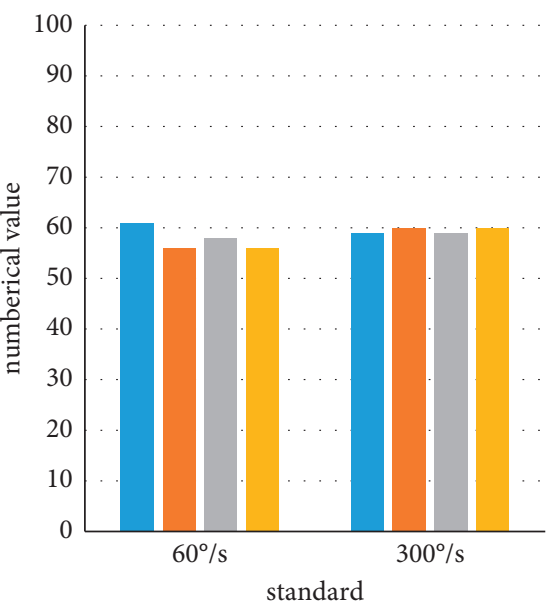

Excellent left Excellent right Normal left $\quad$ Normal right

Figure 8: The peak moment angle of the knee flexors of male Mountain bikers in the excellent and ordinary groups.

TABLE 7: The average power of the knee joint flexors and extensors of male Mountain bikers in the excellent and ordinary groups (W).

\begin{tabular}{|c|c|c|c|c|}
\hline & \multicolumn{2}{|c|}{$60^{\circ} / \mathrm{s}$} & \multicolumn{2}{|c|}{$300 \% \mathrm{~s}$} \\
\hline & Flexors & Extensors & Flexors & Extensors \\
\hline Excellent group left & $158.47 \pm 61.92$ & $240.68 \pm 87.63$ & $211.20 \pm 28.59$ & $345.01 \pm 72.15$ \\
\hline Normal group left & $99.51 \pm 16.63$ & $165.63 \pm 28.13$ & $205.45 \pm 47.47$ & $310.52 \pm 65.68$ \\
\hline Excellent group on the right & $166.35 \pm 72.89$ & $244.95 \pm 65.26$ & $225.10 \pm 26.47$ & $332.29 \pm 60.36$ \\
\hline Normal group right & $105.46 \pm 16.03$ & $160.97 \pm 24.16$ & $212.50 \pm 39.41$ & $306.13 \pm 53.18$ \\
\hline
\end{tabular}



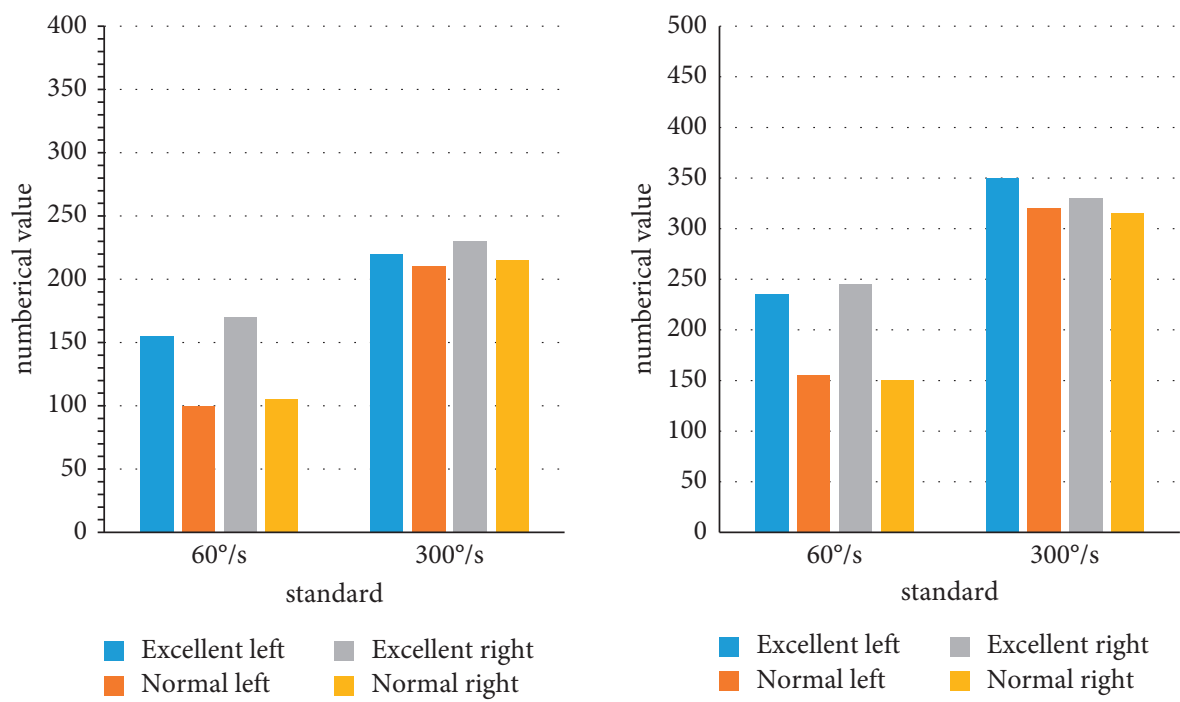

Figure 9: The average power value of the knee flexor group of male Mountain bikers in the excellent and ordinary groups.

TABLE 8: The average power value of the knee flexor group of male Mountain bikers in the excellent group and the general group (W).

\begin{tabular}{lcccr}
\hline & & $60 \%$ s & & $300^{\circ} / \mathrm{s}$ \\
& Flexors & Extensors & Flexors & Extensors \\
\hline Excellent group left & $2.14 \pm 0.66$ & $3.26 \pm 1.06$ & $2.72 \pm 0.55$ & $4.52 \pm 0.85$ \\
Normal group left & $1.29 \pm 0.25$ & $2.15 \pm 0.27$ & $2.72 \pm 0.42$ & $4.15 \pm 1.03$ \\
Excellent group on the right & $2.24 \pm 0.59$ & $3.32 \pm 1.17$ & $2.94 \pm 0.30$ & $4.31 \pm 0.74$ \\
Normal group right & $1.35 \pm 0.19$ & $2.07 \pm 0.33$ & $2.79 \pm 0.53$ & $4.07 \pm 0.81$ \\
\hline
\end{tabular}
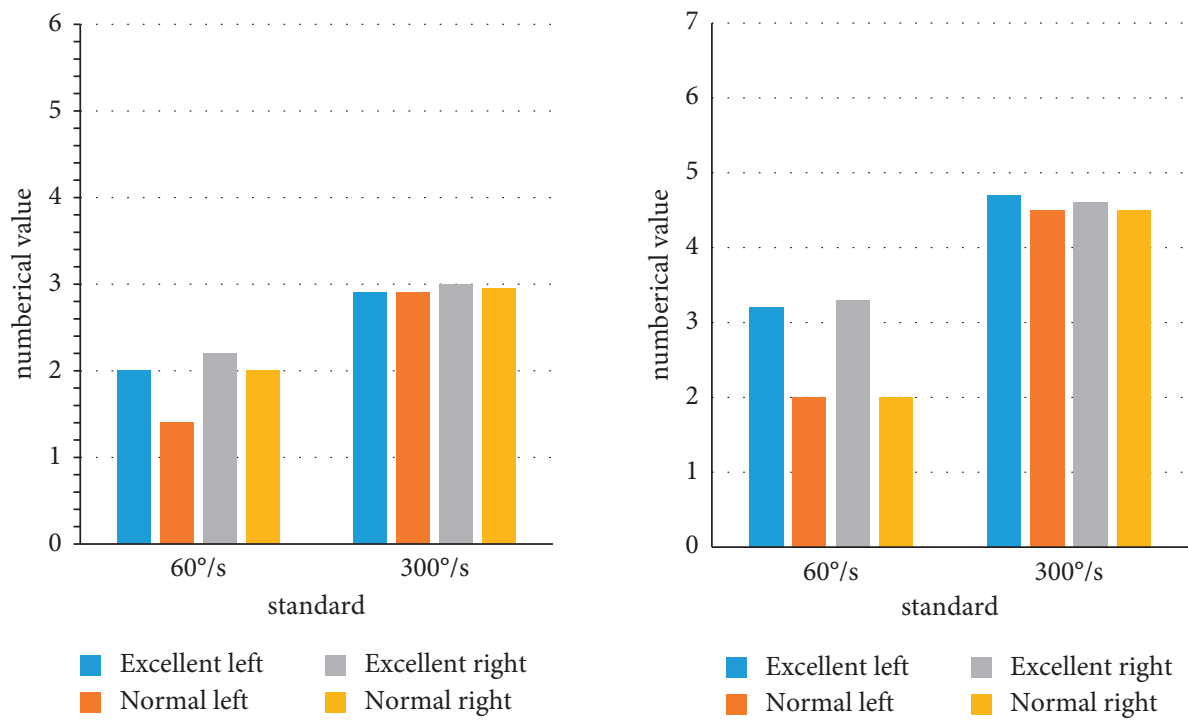

FIGURE 10: The relative average power value of the knee joint extensor group of male Mountain bikers in the elite group and the general group. 
performance. But, having a good functional state may not necessarily be able to achieve good athletic performance. Therefore, a good state of function is one of the prerequisites for good athletic performance.

\section{Discussion}

The joint is an elliptical flexor joint consisting of a femoral tibial joint and a femoral femoral joint. It is attached with a meniscus to strengthen and prevent the movement of the knee joint. Therefore, the knee joint has become the most complex part of the human body. There are many structures that support it. It is located between the two highest lever arms of the human body and is easy to be found when carrying goods and participating in sports. The exercise of the knee flexors is related to the position of the hip muscles. If the knee joint is fixed at a close distance or a long distance, the knee joint can be moved. The main muscles that make up the knee flexors are semitendinosus, semimembranosus, and biceps femoris. The main muscle that lengthens the knee joint is the quadriceps, which stretches the calf at the knee joint during fixation. Judging from the literature of domestic and foreign research reports, the most common examination for clinical diagnosis is the isokinetic muscle of the knee joint. The knee joint, as a stressed part, is easily injured [22]. In addition, the integrity of the knee joint is reinforced when testing the knee joint, the reliability of the test result is higher, the torque change is clear, and the judgment is easier. Also, it is related to the more complete fixation during the knee joint test, the higher reliability of the test results, the clearer torque curve, and the easier judgment. The experiment selected peak moment, relative peak moment, flexionextension ratio, peak moment angle, average power, and relative average power as observation indicators. Different indexes can, respectively, represent the strength indexes, balance indexes, and work indexes of knee joint flexors and extensors. This paper uses the isokinetic strength test rehabilitation system to test and analyze 12 mountain bike athletes above level 1 and 16 mountain bike athletes below level two. This article summarizes the knee agility and muscle strength of the excellent group (above level one) and the general group (below level two). It is particularly important for guiding cyclists to train and prevent knee joint injuries. As the test speed angle increases, this article analyzes the peak torque of mountain bike athletes' knee flexion and extension. This is consistent with previous research results on the characteristics of sports anatomy and muscle mechanics, as well as the theory of Hill's classical equations, in which muscle tension is inversely proportional to muscle contraction rate. The maximum torque refers to the maximum torque output produced by a muscle or a group of muscles as traction during the joint movement of the muscle. It can actually display the maximum muscle load during exercise, representing the largest muscle mass in a group of muscles or joint muscles. When the measured angular velocity is $60 \%$, the dynamic level of knee muscle force is the highest. When the angular velocity is $300 \%$, the strength of the knee joint and the degree of rapid muscle contraction can be tested. The test results show that the maximum strength training should be improved when the test torque value is $60 \%$ s. Maximum strength training is carried out on the basis of intensity training, which can increase the athlete's riding speed without increasing muscle mass. The riding speed of mountain bikes is limited by fast power. Fast power is a special power quality that combines power and speed, and is an integrated part of power and speed. The rapid increase in strength not only increases the speed of muscle contraction but also increases the maximum amount of strength. The greater the energy consumed by the athlete to complete the action and the faster the speed, the greater the energy displayed. The use of high-intensity and slow training methods can promote the rapid transfer of muscle balls and actin filaments, as well as the rapid contact after the next reduction [23].

The ratio of the knee flexion torque to the extensor muscles of mountain bikers above and below the second level increases with the increase of angular velocity. It shows that the knee flexors and extensors tend to be more aligned and relatively balanced. The ability to coordinate and balance plays an important role in cycling (rotating the highest point by 0 degrees and the lowest point by 180 degrees). If the flexor muscles are not well coordinated, it will not only produce a lifting force but also produce negative air when stretching the flexors and extensors. Therefore, while exercising the strength of the knee flexors, we must not forget to improve the coordination ability of the extensors and flexors.

The average power and relative average power of the ankle dorsiflexor plantar flexor group of male mountain bikers increase with the increase of the test speed. This is consistent with previous studies. The average power of the gas increases significantly with the increase of the test speed. This is the result of rapid muscle exertion, and this law is also a sport characteristic of mountain biking [24]. It shows that the ankle dorsiflexion and plantar flexion muscles of mountain bikers have a strong ability to adapt to fast movements. The average power of the ankle dorsiflexor of male mountain bikers above level 1 is significantly greater than that of male mountain bikers below level two. The relative average power between the left and right ankle dorsiflexion and plantar flexion of male mountain bikers above and below the second level is basically balanced. This also ensures that the cyclist can complete the pedaling and lifting action in a short time.

\section{Conclusion}

The functional test index system for mountain bikers is a systematic project. The relationship between functional indicators is dialectically unified; acid and nitrogen kinase can cause blood urea to increase. After two high-load training sessions, serum testosterone decreased and cortisol increased. The changes in these indicators are what we expect to see. Due to the different training conditions of different athletes, individual indicators may not reach the abovementioned changes. Therefore, the changes in the indicators cannot reflect the real state of the athletes. Various indicator data are only relative. As for the situation, we 
cannot blindly maintain high or low values. Hemoglobin is usually formed within 12-24 hours, creatine kinase is formed within 36-72 hours, and urea nitrogen in the blood is formed within 24-36 hours. The high muscle strength of the right and left extensors and right flexors of mountain bikers below the second level still needs to be improved. The extensor muscles of the left and right knees of mountain bikers below grade 2 are not well developed. It needs to strengthen the right knee joint extensor training and correct the uneven pedaling as soon as possible. Mountain bike athletes above and below the second level have no activity in the knee joint extensors and need to strengthen the continuous strength training of the knee joint extensors. Mountain bikers below the second level will have ankle dorsiflexor plantar flexor imbalance, and the maximum strength training of the right ankle dorsiflexor plantar flexor should be improved. Mountain bikers below the second level should focus on the development of high-intensity ankle dorsiflexors and fast-paced strength training. The explosive power of the knee joints and pelvis of mountain bikers below level one is better than that of mountain bikers below level two. The flexibility and stretchability of the knee and ankle joints and flexion joints of mountain bikers above level 1 are slightly higher than those of mountain bikers below level two. Mountain bikers in the second level need timely and targeted training. If it wants to improve muscle strength training, it must increase the strength of training flexors. It needs to increase the proportion of the athlete's pelvic floor and improve the coordination of the flexors of the knee and ankle joints. The experiment has discussed whether the mountain bike rider's knee joint, flexion-extension joint bone, and the ratio of flexion-extension are reasonable through analysis. It is an important factor in becoming an excellent athlete. Therefore, it is recommended that the majority of mountain bikers strengthen special training, improve the working ability and performance characteristics of knee joints and extensors, and improve energy supply and metabolism, so that they can quickly become better athletes. The sample in this study is small and needs to be expanded in the future. The relationship between the strength of the knee and ankle joint flexors and extensors at different angular velocities and the optimal range of ratios need to be further explored. If there is anything wrong with the above conclusions, please criticize and correct them, and jointly contribute to improving the level of cycling.

\section{Data Availability}

No data were used to support this study.

\section{Conflicts of Interest}

The author declares that there are no conflicts of interest.

\section{References}

[1] C. Lu, S. Wang, and X. Wang, "A multi-source information fusion fault diagnosis for aviation hydraulic pump based on the new evidence similarity distance," Aerospace Science and Technology, vol. 71, pp. 392-401, 2017.

[2] C.-Q. Zhang, P.-K. Chung, and G. Si, “Assessing acceptance in mindfulness with direct-worded items: the development and initial validation of the athlete mindfulness questionnaire," Journal of Sport and Health Science, vol. 6, no. 3, pp. 311-320, 2017.

[3] J. D. Kurtz, R. J. Kanter, M. Olen, and A. F Rossi, “Screening the apparently healthy athlete for risk: a paradigm in transition," Cardiology in the Young, vol. 27, no. S1, pp. S89-S93, 2017.

[4] J. Gallagher, I. Needleman, P. Ashley, R. G. Sanchez, and R. Lumsden, "Self-reported outcome measures of the impact of injury and illness on athlete performance: a systematic review," Sports Medicine, vol. 47, no. 7, pp. 1335-1348, 2017.

[5] L. J. Weiss, D. Wang, M. Hendel, P Buzzerio, and S. A Rodeo, "Management of rotator cuff injuries in the elite athlete," Current Reviews in Musculoskeletal Medicine, vol. 11, no. 3, pp. 102-112, 2018.

[6] M. J. Konings and F. J. Hettinga, "Objectifying tactics: athlete and race variability in elite short-track speed skating," International Journal of Sports Physiology and Performance, vol. 13, no. 2, pp. 170-175, 2018.

[7] C. Granier, C. R. Abbiss, A. Aubry et al., "Power output and pacing during international cross-country mountain bike cycling," International Journal of Sports Physiology and Performance, vol. 13, no. 9, pp. 1243-1249, 2018.

[8] C. Sánchez-Muñoz, J. J. Muros, and M. Zabala, "World and Olympic mountain bike champions' anthropometry, body composition and somatotype," The Journal of Sports Medicine and Physical Fitness, vol. 58, no. 6, pp. 843-851, 2018.

[9] M. S. Kuhar, "From quarry to mountain bike park," Rock Products, vol. 121, no. 6, p. 72, 2018.

[10] L. Engelbrecht and E Terblanche, "Physiological performance predictors in mountain bike multi-stage races," The Journal of Sports Medicine and Physical Fitness, vol. 58, no. 7-8, pp. 951-956, 2018.

[11] B. Wei, "Carbon fiber: not just for your mountain bike anymore," The Journal of Thoracic and Cardiovascular Surgery, vol. 156, no. 4, pp. e181-e182, 2018.

[12] M. J. Winston and A. J. Weiland, "Scaphoid fractures in the athlete," Current Reviews in Musculoskeletal Medicine, vol. 10, no. 1, pp. 38-44, 2017.

[13] T. S. Kawauchi, I. Umeda, L. M. Braga et al., "Is there any benefit using low-intensity inspiratory and peripheral muscle training in heart failure? A randomized clinical trial," Clinical Research in Cardiology, vol. 106, no. 6, pp. 764-765, 2017.

[14] G. D. Rodrigues, J. L. Gurgel, T. R. Gonçalves, and P. P da Silva Soares, "Inspiratory muscle training improves physical performance and cardiac autonomic modulation in older women," European Journal of Applied Physiology, vol. 118, no. 12, pp. 1143-1152, 2018.

[15] R. Peebles and C. E. Jonas, "Sacroiliac joint dysfunction in the athlete," Current Sports Medicine Reports, vol. 16, no. 5, pp. 336-342, 2017.

[16] R. Ueno, K. Sakanaka, S. Ikeda, and Y. Horiguchi, "Purification of a pepstatin insensitive protease from mackerel white muscle*1," Nippon Suisan Gakkaishi, vol. 54, no. 4, pp. 691-697, 2017.

[17] S. L. Domazet, A. Grøntved, T. K. Jensen, N. Wedderkopp, and L. B. Andersen, "Higher circulating plasma polychlorinated biphenyls (PCBs) in fit and lean children: the European youth heart study," Environment International, vol. 136, no. 2, pp. 90-94, 2020.

[18] M. Miyazaki and K. A. Esser, "Cellular mechanisms regulating protein synthesis and skeletal muscle hypertrophy in animals," Journal of Applied Physiology, vol. 106, no. 4, pp. 1367-1373, 2009. 
[19] B. Gaffney and B. M. Gaffney, "Digital commons @ du evaluation of novel high-density emg feedback parameters on the spatial distribution of trapezius muscle activity evaluation of novel high-density emg feedback parameters on the spatial distribution of trapezius muscle activity," European Journal of Applied Physiology, vol. 102, no. 3, pp. 325-333, 2019.

[20] W. M. Akkila, R. L. Chambers, O. I. Ornatsky, and J. C. McDERMOTT, "Molecular cloning of up-regulated cytoskeletal genes from regenerating skeletal muscle: potential role of myocyte enhancer factor 2 proteins in the activation of muscle-regeneration-associated genes," Biochemical Journal, vol. 325, no. 1, pp. 87-93, 2019.

[21] M. Dres, B.-P. Dubé, J. Mayaux et al., "Coexistence and impact of limb muscle and diaphragm weakness at time of liberation from mechanical ventilation in medical intensive care unit patients," American Journal of Respiratory and Critical Care Medicine, vol. 195, no. 1, pp. 57-66, 2017.

[22] K. Li, Y. Wang, A. Zhang, B. Liu, and L. Jia, "miR-379 inhibits cell proliferation, invasion, and migration of vascular smooth muscle cells by targeting insulin-like factor-1," Yonsei Medical Journal, vol. 58, no. 1, pp. 234-240, 2017.

[23] M. Abe, G. Murakami, M. Noguchi, S. Kitamura, K. Shimada, and G. I. Kohama, "Variations in the tensor veli palatini muscle with special reference to its origin and insertion," The Cleft Palate-Craniofacial Journal, vol. 41, no. 5, pp. 474-484, 2017.

[24] Q. Zhang, J. Zheng, J. Qiu et al., “ALDH2 restores exhaustive exercise-induced mitochondrial dysfunction in skeletal muscle," Biochemical and Biophysical Research Communications, vol. 485, no. 4, pp. 753-760, 2017. 